\title{
DIABETES VS. PHYSICAL EXERCISE
}

\author{
Diabetes vs. physical exercise
}

BARBARA RACZYŃSKA', ŁUKASZ ZUBIK', MICHAŁ JELIŃSKI ${ }^{2}$

\section{The Josef Pilsudski Uniwersity of Physical Education in Warsaw, Faculty of Physical Education and Sport in Biata Podlaska, Department of Physiology \\ 2 Polish Association of Rowing Clubs}

\author{
Mailing address: Barbara Raczyńska, Faculty of Physical Education and Sport, 2 Akademicka Street, \\ 21-500 Biała Podlaska, tel.: +48 83 3428827, fax: +48 83 3428800, \\ e-mail: barbara.raczynska@awf-bp.edu.pl
}

\begin{abstract}
Diabetes is a disease affecting people of all races in the world, it can appear at any age. It is considered to be social disease due to the incidence and complications. It is a disease of complex etiology, classified as a metabolic disease with chronic hyperglycemia. It requires intensive hypoglicemizing therapy. There are two types of diabetes: type I and type II with different etiologies and varied clinical picture.

In the case of prolonged illness serious complications develop, from which, however, the sick may be saved, on condition of the patient's absolute submission to the rigors of treatment. It is possible to live an active life and even practice sports. Extremely important in this case is the correct blood glucose, providing for adjusted glucose homeostasis, which is promoted by physical activity. Physical exercise can be an important therapeutic agent in treating diabetes, provided the criteria of its physiological tolerance are determined. It is vital that an individual approach to the patient is made, associated with the duration of the disease and existing complications. Patient education in monitoring patients' glucose levels is important, i.e. the efficiency in the implementation of individual insulin therapy and hyperglycemia or hypoglycemia self-prevention during and after physical exercise. Patients who decide to practice sport should find the optimal way to control exercise, and diet during and after exercise, then the sporting success is possible.
\end{abstract}

Key words: diabetes, exercise, blood glucose

\section{Introduction}

Diabetes is a disease affecting people of all races throughout the world, it can appear at any age. It is considered to be social disease due to the incidence and complications. It represents $95 \%$ of the total carbohydrate metabolic disorders resulting from the total lack of insulin or insulin resistance. According to the World Health Organization (FAO), diabetes affects around 100 million people in the world, in Poland about $3 \%$ of the population (2.0 million), and the number of people with impaired fasting glucose or glucose tolerance is about twice as that. Elusive onset of the disease is found in over $85 \%$ of cases [1,2].

Diabetes is a disease of complex etiology classified as metabolic diseases with chronic hyperglycemia. There are two types of diabetes of different pathophysiology: type I, resulting from autoimmune damage to pancreatic islet Bcells in individuals genetically predisposed and type II - in its creation a key role is played by genetic predisposition, associated with impaired insulin secretion and tissue resis- tance to its action $[3,4]$. In the case of prolonged illness there develop severe organ complications (macro-and micro-angiopathy - changes in the vessels and peripheral nerves), associated with prolonged hyperglycemia. The clinical picture of these disorders is very diverse, due to the concomitant disturbances of fat tissue, protein and hydromineral (transport electrolytes) management $[5,6,7]$. It is necessary to undergo intensive glucose lowering therapy throughout the patient's life. However, it is possible to save patients from complications (atherosclerosis, stroke, retinopathy, nephropathy), they may lead a long active life and is not impossible for them to participate in sports, based on the patient's absolute submission to the rigors of treatment.

\section{Insulin deficiency and excess}

Regulation of insulin secretion is executed by feedback via a direct effect of blood glucose on B-cells of pancreatic islets. When the concentration is high (above $300 \mathrm{mg} / \mathrm{dl}$ ) 
insulin secretion is increased, but if it is correct (70-120 $\mathrm{mg} / \mathrm{dl}$ ) or decreased rate of secretion is small $[8,9]$.

The main feature of insulin deficiency is elevated levels of blood glucose due to its decreased penetration into many tissues, and increased release of glucose from the liver (excess glycogen). The resulting hyperglycemia leads to glycosuria and osmotic diuresis causing dehydration, accompanied by a loss of $\mathrm{Na}+$ and $\mathrm{K}+$. In extreme cases, these disorders lead, through toxic effects on the nervous system, to coma and death [8].

The excess of insulin and the resulting shortage of blood glucose lead to hypoglycemia. In the absence of the basic material of the energy system which is glucose, the body's energy needs can be met through the degradation of protein and fat reserves. This leads to their increased catabolism, in consequence the increased protein degradation (negative nitrogen balance) and loss of muscle mass, and in case of fats breakdown there occurs the accumulation ketone bodies in the blood (ketosis) - metabolic acidosis develops. If hypoglycemia is extended, irreversible changes occur in the structure of the brain, the diencephalon and medulla oblongata. It is followed by respiratory depression and death.

\section{Carbohydrate metabolism}

Carbohydrates distributed in the tissues of plants and animals are an important source of energy for life processes activity. The most important sugar serving as metabolic fuel of the tissues is blood glucose. Glucose homeostasis is the result of glucose supply into the blood and its consumption in the cells. Constant concentration of glucose in the blood is dependent on the balance of these processes, regulated by the liver, acting as a kind of "glucostat".

The main determinants of blood glucose levels are: food intake, rate of penetration of glucose into myocytes, fat cells and other organs, and glucostatic liver activity. Five percent of ingested glucose is immediately converted into glycogen, and $30-40 \%$ into fats. Remainder is metabolized in muscle and other tissues and metabolic processes are regulated by the concentration of substrates and hormones. In addition to insulin, metabolism involves many hormones in the regulation of glucose, they also include glucagon, epinephrine, norepinephrine, glucocorticoids, growth hormone and thyroid hormones, and many other factors, including genetic factors and lifestyle $[9,10,11]$.

The transport of glucose into cells is mediated by specific carrier proteins: GLUT1 to GLUT5 and GLUT7, the most active protein is a transporter GLUT4 [12]. Transport is regulated by two distinct mechanisms: insulin dependent (skeletal muscle, liver, adipose tissue), and independent of insulin (brain, red blood cells, but also the tissues listed above). Decisive influence on glucose metabolism in humans, the resting level and stimulated by insulin use, exert skeletal muscles, due to their mass [13, 14]. All types of muscle fibers are sensitive to insulin, although the red fi- bers have a greater number of insulin receptors and glucose transporters (GLUT-4) than white [15]. Muscle spasm is associated with an increase in biological diffusion of glucose from the blood and the extracellular space into the working muscle cells, independently of insulin, and increased sensitivity of muscle cells to insulin. Processes are accompanied by a reduction in insulinemia, which allows to conserve glucose $[15,16]$. Studies have shown that an important controller in glucose metabolism and an important factor in the maintenance of glucose homeostasis, in addition to a balanced diet, is the physical exercise, but the effect is short-term (2-4h). However, the increase of myocytes sensitivity to insulin was observed for another 48 hours after exercise $[14,17,18,19]$. Studies have shown that only the constant, regular physical activity is beneficial for glucose tolerance and its distribution in the body [20, 21]. These observations became the basis for the systematic use of physical exercise in the treatment of diabetes, as supporting the maintenance of glucose homeostasis (normoglycemia) $[15,16]$.

\section{Hormonal regulation of glucose level}

Maintenance of normoglycemia during exercise in healthy individuals is largely through the adaptation hormone. Each of the hormones involved in carbohydrate metabolism plays an important role in providing adequate quantities of energy to working muscles. And so the exercise enhances the activity of glucagon, and catecholamines resulting in increased lipolysis and release of free fatty acids (FFA) from adipocytes, as feedstock in the process of gluconeogenesis in the long lasting exercises. A similar role is attributed to the increase in epinephrine under the influence of exercise [2]. FFA consumption as the energy material is enhanced by hormones such as glucocorticoids (cortisol) and growth hormone, whose synthesis during exercise also increases [23]. Effect of physical exercise is therefore associated with the production, flow and use of substrates; hypoglycemic effect (increase in glucose uptake), and the hyperclycemizing effect of by increased gluconeogenesis (release of FFA, alanine and lactate from the muscles).

This well-coordinated hormonal response of the body to the physical exercise, conditioning the maintenance of glucose homeostasis, disappears in patients with insulin deficiency, mainly in patients with type I diabetes, which can be prevented by monitoring the glucose levels during exercise, and adequate nutrition [8, 24, 25].

\section{Glucose level vs. physical exercise}

In healthy people exercise leads to changes in blood glucose levels, which depend on the intensity and duration. During short-term exercises exercise-dependent increase in glucose level is observed, indicating glucose production advantage over its use, the consumption of glucose increases with the intensity of exercise. During the exercises of 
moderate intensity and longer duration glucose concentration begins to decrease to a level at which symptoms of hypoglycemia occur (the long-term exercises) [26].

Increased glucose production is the result of glycogenolysis and gluconeogenesis increase in the liver. In the early stage of exercise, small gluconeogenesis is involved. With extension of working time intensity of this process increases, the supply of substrates to the liver for the synthesis of glucose increases, that is: FFA, glycerol, amino acids, mainly alanine, as well as pyruvate and lactate.

In diabetic patients exercise-related blood glucose changes also depend on the intensity and duration of exercise, but the reactions are different. In patients with severe insulin deficiency the exercise often leads to increased hyperglycemia, because participation of gluconeogenesis in the process is greater than in healthy subjects. The reaction is not only a result of insulin deficiency, but probably greater than normal activation of factors that stimulate the production of glucose. In diabetic patients there is also noted an increase in ketone bodies, which during exercise may be significant, indicating the advantage of their production over consumption (increased levels of FFA in the blood flowing to the liver) [16]. In case of patients treated with insulin the exercise generally leads to reduction of blood glucose, as a result of peripheral glucose uptake. In addition, exogenous insulin may inhibit the release of glucose from the liver. However, during and after exercise there may occur a state of hypoglycemia, which usually occurs in the fasting state, when the liver is the only source of glucose. In such circumstances, the patient must eat the right amount of rapidly absorbed carbohydrates (food glucose, sugar, sweet juice) to compensate for glucose [27].

The conversion of carbohydrates in the working muscles is linked to lactate production, which in healthy people is proportional to the intensity of exercise. However, in patients with diabetes increase of lactate levels may be higher after exercise, and the duration of the increased concentration after exercise longer, due to inhibition of removal of lactate from the blood by glucose-lowering drugs (insulin, biguanidin and others) [16].

It is believed however, that the appropriately dosed muscle work positively affects the normalization of blood glucose, the health status of patients with diabetes, provided continuous monitoring of the intensity and duration. Working muscles can utilize glucose with the minimum concentration of insulin in the blood reducing the demand for this hormone. An important problem is to determine the physiological criteria for exercise tolerance in patients with diabetes, the other for people with type I diabetes, the other for patients with type II. It becomes essential to consider the benefits and possible risks of use of exercise and how to prevent them $[28,29,30]$.

\section{Tolerance of physical exercise in diabetes}

Tolerance of physical exercise by people with diabetes is reduced by the associated with the disease, regulation disorders (neuro-autonomous), insulin deficiency, the influence of hypoglycemic drugs and others (in the case of accompanying diseases), the effect of diet modification (low carbohydrate diet), syndromes of complications. It is necessary, prior to physical therapy, to determine an individual exercise tolerance associated with the duration of illness, type of diabetes, treatment regimens, including place and way of insulin administration, as well as age and sex.

In addition, there are a number of other elements affecting the exercise tolerance, among them its intensity, metabolic rate, the personal degree of fitness and time relationship of exercise with meals. Exercises by patients with type I diabetes are linked more often than in patients with type II diabetes, to the risk of violent disturbances of homeostasis in the form of hypo- or hyperglycemia, which may lead to severe complications of the circulatory system $[31,32]$.

Before commencement of the training session, therefore, assessment of the circulatory system is needed in consideration of the possibility of vascular complications, and the evaluation of the nervous system and eyes, as well as identification of comorbidities [7]. The basic condition for physical activity taking by people with diabetes is aligned with the metabolism of glucose, i.e. if blood glucose levels do not exceed $250 \mathrm{mg} / \mathrm{dl}(13.9 \mathrm{mmol})$. The concentration of glucose before exercise may also be lower than $100 \mathrm{mg} / \mathrm{dl}$ $(5.5 \mathrm{mmol})$. In patients treated with insulin may need to reduce the dose of insulin depending on the planned exercise intensity and duration or the introduction of additional portions of carbohydrates [19, 22].

At the time of preparation of an individual program of exercises, which are primarily aimed at the therapeutic effect it is necessary to comply with certain recommendations [16].

Exercise load of people with uncomplicated type I diabetes should not exceed $30 \%$ of their physical capacity, even if it is a short-time exercise [30]. Patients with diabetes should not perform static exercises because of an increase in blood pressure. Anaerobic exercises are not recommended, as well as maximal and supramaximal, also significantly raising blood pressure (stimulating the adrenergic system). The training programs use moderate therapeutic aerobic exercises, increasing the size of maximum oxygen consumption $\left(\mathrm{VO}_{2} \mathrm{max}\right)$, the efficiency of lung ventilation, cardiovascular endurance, as well as neural adaptation and increase in the amount of muscle tissue. The result of exercises carried out regularly, also involves loss of excess body weight (primarily body fat) important for people with type II diabetes, which is associated with improved insulin sensitivity, and normalization of blood glucose and serum lipids. These benefits are associated with delayed occurrence (or non-occurrence) of organ complications of the 
disease, which are the main cause of disability or premature sudden death. One also cannot forget the positive impact of exercise on patients' mental health [quoted after 6].

As recommended by the American College of Sports Medicine, people with diabetes should perform 3 to 5 times a week a 20-60 minutes aerobic exercise training at 55-60\% maximum heart rate (HR). For those with excess body fat it is recommended to extend the workout to one hour while reducing its intensity [33].

\section{Principles of physical training organization}

Detailed rules for the organization of therapeutic physical exercise recommended to the patient with diabetes, as well as absolute and relative contraindications to its use, have been provided by Bernas [16]. The most important absolute contraindications are: complicated recent heart attack, unstable angina, acute heart failure, phlebitis, acute renal failure. The relative contraindications include: blood glucose levels above $250 \mathrm{mg} / \mathrm{dl}$, acetonuria, tachycardia, hyperthyroidism, AIDS.

Prelude to the implementation of the training involves the classification by the physician covering the identification and assessment of vascular or neurological complications, ECG evaluation and diagnosis of diseases associated with diabetes. It is also recommended to examine pressure response to exercise (can be increased).

\section{Type of exercise}

In people with diabetes aerobic and dynamic exercises are preferable. Among the exercises the following are recommended: tourism, walking, slow running (jogging), swimming, badminton, playing football, cycling, gymnastics [34]. All the exercises that may cause injury should be excluded.

In general, in addition to the simplest form of exercise, i.e. walking, the following exercises are recommended:

- general improving exercises with elements of respiratory gymnastics, 20-30 minutes,

- physical games and some elements of sports, fitness trails - a combination of walking, gymnastics and sports items to 60 minutes,

- individual exercise dosed on the cyclo-ergometer at home (once a day for 20-30 $\mathrm{min}$ ),

- team or individual exercises in water (60 minutes),

- treatment by physical work (work on the plot),

- jogging - less intensive run, rather walking-running, time limited by the ability of the patient, to 60 minutes.

Prior to practice in the field or the gym, a 5-10 minute warm-up is applied, whose aim is to gradually introduce the patient to adapt to a full exercise. These are usually light, dynamic exercises of stretching, warming up muscles, such as bending [16, 35].

\section{Exercise intensity}

After warming up, the intensity of exercise is adapted to the individual patient's physical capacity. It is determined mostly by an indirect method calculating the maximum oxygen uptake $\left(\mathrm{VO}_{2} \mathrm{max}\right)$, based on post-workout assessment of pulse rate (HR), for example by Astrand and Ryhming method [36]. Exercises recommended are at the level of $30-50 \% \mathrm{V0}_{2}$ max. During the exercise the heart rate should be controlled, blood glucose, blood pressure, which should not rise above $180 / 95 \mathrm{mmHg}[35,37]$.

In order to select the appropriate exercise intensity, the following should be determined:

1) pulse rate at rest - in the morning in lying position, after waking up - HR at rest,

2) the maximum pulse rate - HRmax, which can be calculated according to the latest recommendation, using the formula: $\mathrm{HR}_{\max }=208-0.7 \mathrm{x}$ age of the patient in years [38],

3) then $50 \%$ of maximum efficiency (ME50) of the person examined is calculated according to the following formula:

$$
\mathrm{ME}_{50}=0,5\left(\mathrm{HR}_{\max }-\mathrm{HR}_{\text {at rest. }}\right)+\mathrm{HR}_{\text {at rest. }}
$$

\section{Exercise duration and frequency}

Exercises at ME50, can safely take 20 to 45 minutes, those with lower intensity may take longer. It is believed that shorter exercises do not give the expected metabolic effects. Tangible benefits in terms of increased insulin sensitivity and decrease in glucose levels are achieved by conducting exercises every second day, and at least three times a week. Post-physical exercises improvement of blood glucose generally takes 12 to 72 hours [16].

At the completion of exercises, for 5-10 minutes the patient should perform exercises with decreasing intensity (i.e. hypothermia after exercise).

It is important for practitioners that their blood glucose is monitored frequently, especially at the beginning of the training program.

The benefits of regular exercise are less pronounced in people with type I diabetes than type II. This is mainly due to less frequent lipid abnormalities in patients with type I diabetes.

Although exercise is an important method of treatment for diabetes, but not everyone can make it to the full extent, which is generally subject to the duration of existing diseases and complications. As mentioned above, an individual approach to the patient is important. However, the extension of daily physical activity is necessary. The first phase of increased activity consists of: 10-15 minutes of gymnastics in one's own apartment, a walk (3-4 times per week) (it can be marching but not necessarily) and also a reduction, if possible, of using elevators, car [8].

In the second stage it is necessary to introduce physical activity into lifestyle. This applies to any kind of physical 
exercise 3-5 times a week (30 minutes), brisk walking, cycling, swimming and team sports: basketball, volleyball, tennis are recommended.

This does not exclude also professional sport with the consent of doctor, after making appropriate clinical studies. Patient joining the sport must be properly trained in this direction. He should know the side effects and contraindications of exercise [39]. Patient education in glucose monitoring is very important, which is an essential element of the treatment process, as well as sports training.

In any case, prior to the training there is a requirement of an initial assessment of metabolic status and degree of glycemic control and performance of individual patient evaluation in insulin therapy, his ability of glycemic selfcontrol and hyper- or hypoglycemia self-prevention after the exercises [40].

The increasing incidence of young people, including children and young people is a major problem in diabetes therapy. This applies particularly to people who are physically active, often practicing different sports. Also a fashion for extreme sports, which prevailed in Europe, and Poland is a challenge for people with diabetes, but also for medical care. Patients, particularly teenagers, want to catch up with healthy peers in the sport, but often are not aware of the dangers faced during exercise, and during post-exercise period, which can result in serious complications.

So, can patients with diabetes practice sports?

It is a difficult question, requiring big commitment of both the patient and the physician. Huge advance in knowledge and better understanding of pathogenic mechanisms of complications give hope to increase of the effectiveness of treatment of diabetes. They make it possible to monitor it better and better, even under conditions of stress. An example is the use of individual pump. Continuous subcutaneous insulin infusion implemented by pump mimics the most physiological rhythm of insulin secretion by a healthy pancreas. It ensures optimal metabolic control, as it creates the possibility of better control (than a single injection) of the basic and postprandial insulin levels [41].

There is the possibility of providing the patient with accurate and practical tips on how to adjust insulin therapy to the individual needs of the body during exercise without having to sacrifice the sport. This implies, however, an extensive education conducted in the diabetes clinic, where accurate, practical guidelines for drug therapy, and diet during therapeutic exercise and sport are provided [23].

\section{Diabetes vs. sport}

Patients who decide to practice sport in addition to fighting with their own weakness, related to the disease, must confront the danger of losing consciousness due to hypoglycemia after physical exercises. Sport practicing must therefore be very balanced, which applies both to patients with type I and type II diabetes, since the patient is responsible for acute complications caused by exercise, who, after proper training conducts his own treatment. There is no recipe for insulin therapy during exercise. It is the patient, who by trial and error by doing 8-12 measurements of the sugar level (a day), should find the optimal way of controlling exercise, and diet during and after exercise $[23,25]$.

Patients treated with insulin cannot affect the level of exogenous insulin during exercise. They have a tendency to hypoglycemia during and after exercise. The patient must be prepared for the possibility of hypoglycemia and be able to prevent it, and when there is an appropriate response (consumption of easily digestible simple carbohydrates). In most cases, hypoglycemia is relieved after the administration from 10 to 15 grams of rapidly absorbed carbohydrates (15 minutes). Then again, blood glucose levels should be checked (it should not be below $70 \mathrm{mg} / \mathrm{dl}$ ). In the case of severe hypoglycemia athlete should be given glucagon (medical assistance). Risk of post-exercise hypoglycemia is often caused by long-term exercise, in proportion to the duration, and the risk may persist even up to 24 hours. The patient must be aware that during the intensive training period at all times there is at risk of hypoglycemia, which lasts for 1-2 weeks after its completion, which results from sensitization of tissues to insulin and the process of rebuilding glycogen reserves [23]. In the swift recovery of glycogen after exercise carbohydrate intake with high glycemic index plays an important role. It is also important to eat before exercise the additional 2-4 WW (replacements of carbohydrate), and then every 30 minutes to eat $2 \mathrm{WW}$, preferably in the form of an isotonic fluid [28].

Exercise undertaken in a state of insulin deficiency, with high levels of blood glucose (above $300 \mathrm{mg} / \mathrm{dl}$ ) is dangerous, and "sugar reduction" by exercise is not justified, and in this situation there should be a corrective dose of insulin administered or oral medication, increased by 10$20 \%$. Otherwise, the sugar level can be very high, also due to the body's defensive reaction by the stress hormones (adrenaline, cortisol). Acetone may occur due to abnormal metabolism of fat. If ketone is found to be present in the blood, the exercise must be stopped [29, 42].

During the training period, the frequency of measurements of blood sugar levels should be increased. Especially the attention should be paid to the glucose level at 2-3 at night, when patients with diabetes can suffer from postexercise hypoglycemia (quoted after 23). The method of continuous glucose monitoring is very helpful, at least for 3 days, which allows to detect asymptomatic hypoglycemia. In the conditions which prevent control of blood sugar levels, professional sport practicing, especially extreme sport is dangerous in every respect.

An indispensable condition for safe sports practicing is nutrition subordinated to both diabetes and practiced sport. Nutrition must always cover all the body's requirement for nutrients, but must also consider the impact of the products consumed on carbohydrate metabolism, glucose, lipidemia, and other factors (e.g. hydration) in such a way as to be 
able to coordinate drug therapy [25, 43, 44]. It must be adapted to the situation of exercise, training and competition. The diet should take into account certain preferences for both kinds of products, especially their GI. Some differences relate to the abundance of food and frequency of their consumption. It is preferred to consume 5-7 smaller meals per day, which must be tailored to the patient's lifestyle, and medications. Coordination between the injection of insulin (or taking of drugs) and eating a meal and exercise implementation is the guarantor of the balanced glucose level. Dietary recommendations for persons undergoing physical exercise are provided by numerous case studies and brochures, popular science and popular publications that can be found in each diabetes clinic [8, 24, 32].

The balanced blood glucose and safe sports practicing are reflected in medical diagnostic parameters. In addition to monitoring blood glucose, which the patient carries out, it is necessary to periodically check glycosuria in diabetes clinic, acetonuria, and above all glycosylated hemoglobin $\left(\mathrm{HbA}_{1 \mathrm{c}}\right)$, which is a measure of blood glucose-lowering efficacy, because it reflects the average blood glucose over the past 3-4 months [29]. Control periodic medical examination also applies to endurance tests including ECG, and monitoring of possible complications of diabetes.

An example of glycemic control and maintenance of normoglycemia (normal blood $\mathrm{HbA}_{1 c}$ value below $7 \%$ ) during professional sport practicing is the co-author of this article; the lower competing in the heavyweight double four. Skillful adaptation of treatment to the training process (insulin therapy and diet) allowed for safe exercise and gave him sporting success: Olympic gold medal (in Beijing) and 4 World Championship medals.

\section{Literature}

1. Szepietowska, N., Szelachowska M. \& Kinalska J. (2003). LADA - late autoimmune diabetes in adults definition, clinical characteristics. Magazyn Medyczny, Diabetologia 8(5), 20-25. [in Polish]

2. Tatoń, J. (2009). How to develop urgently needed universal and pragmatic program for diabetes control in Poland, increasing the opportunities of obtaining better medico-social results of for 2 million people with diabetes. Med. Metaboliczna 13(4), 9-17. [in Polish]

3. Atkinson, M.A. \& Eisenbarth G.S. (2001). Type I diabetes: new perspectives on disease pathogenesis and treatment. Lancet 358, 221-229.

4. Kinalska, J. (1999). Impaired insulin secretion or insulin resistance. Endokrynologia Polska 50 Supp. 1, 25-28. [in Polish]

5. Zdrojowy, K., Bednarska-Chabowska D. \& Adamiec R. (2003). Macro and micropathy in clinical picture of diabetes. 8(5), 55-61. [in Polish]

6. Zdrojowy, K., Bednarska-Chabowska D. \& Adamiec R. (2003). Macro and micropathies in clinical picture of diabetes. Magazyn Medyczny, Diabetologia VIII (110), 5, 55-61. [in Polish]

7. Zdrojowy, K., Sutkowska E. \& Adamiec R. (2005). Physical exercise and metabolic control. Med. Sportowa 21(5), 370-372. [in Polish]

8. Czech, A., Tatoń J. \& Bernas M. (2000). Compendium of diabetology (pp. 8 A-136). Gdańsk: Via Medica. [in Polish]

9. Zierath, J.R., Krook A. \& Wallberg-Henriksson H. (2000). Insulin action and insulin resistance human skeletal muscle. Diabetologia 43, 821-35.

10. Ganong, W.F. (1994). Physiology. Basics of medical physiology (pp. 407-435). Warsaw: Wydawnictwo Lekarskie PZWL. [in Polish]

11. Tatoń, J. (2003). Cardio-diabetology - the need for intensive cooperation of diabetologists and cardiologists in research and at the patient's bedside. Magazyn Medyczny. Diabetologia 8(10), 17-19. [in Polish]

12. Guma, A., Zierath J.R., Wallberg-Henriksson H. \& Klipa A. (1995). Insulin induces translocation of GLUT4 glucose transporters in human skeletal muscle. Am. J. Physiol. 268, E613-E622.

13. Baron, A.D., Wallace P. \& Brechtel W. (1987). In vivo regulation of non-insulin mediated and insulin mediated glucose uptake by cortisol. Diabetes 36, 12301237.

14. Fink, R.J., Wallace P., Brechtel G. \& Olefsky J.M. (1992). Evidence that glucose transport is rate-limiting for in vivo glucose uptake. Metabolism 41, 867-902.

15. Otto-Buczkowska, E. \& Jarosz-Chobot P. (2001). Effect of physical exercise on glucose homeostasis in people with diabetes. Med. Metaboliczna 2, 37-41. [in Polish]

16. Bernas, M. (2001). Tolerance of physical exercise as a factor in determining the use of physical training therapy in people with diabetes. Med. Metaboliczna 5(3), 57-70. [in Polish]

17. Chang, P.Y., Jensen J., Printz R.L. \& et al. (1996). Over expression of hexokinase II in transgenic mice. J. Biol. Chem. 271, 14834-9.

18. Hjeltnes, N., Galuska D., Bjomholm M. \& et al. (1998). Exercise - indiced over expression of key regulatory proteins involved in glucose uptake and metabolism in tetraplegic persons: molecular mechanism for improved glucose homeostatis. FASEB J. 12, 1701-12.

19. Perseghen, G., Scifo P., De Cobelli F. \& et al. (1996). Increased glucose transport phosphorylase and muscle glycogen synthesis after exercise training in insulin resistant subjects. N. Engl. J. Med. 335, 1357-1361.

20. Lutosławska, G. (2001). Impact of physical exercise on glucose transport to muscles. Med. Sportiva 5(1), 9-15. [in Polish]

21. Vukovich, M.D., Arciero P.J., Kohrt W.M. \& Racette S.B. (1996). Changes in insulin action and GLUT-4 with 6 days of inactivity in endurance runners. J. Appl. Physiol. $80,240-244$. 
22. Carolyn, C.J. (2003). Diabetes vs. exercise. Med. Sportiva 7(1), 49-57. [in Polish]

23. Surdej, B. \& Furgal W. (2006), Physical exercise in patients treated by insulin for diabetes. Med. Sport. Practica 7(2), 22-25. [in Polish]

24. American Diabetes Association. (1998). Nutrition recommendation and principles for people with diabetes mellitus. Diabetes Care 21 (supl. 1), S 32-35.

25. Jarosz, M. (2006). Obesity, Nutrition, Physical Activeness, Health of Poles. Warsaw: IŻŻ. [in Polish]

26. Giacca, A., Groenwoud Y., Tsui E., Mc Cean P. \& Zinman B. (1998). Glucose production, utilization and cycling in response to modern exercise in obese subjects with type 2 diabetes and mild hyperglycemia. Diabetes 47(11), 1763-70,

27. American Diabetes Association Clinical Practice Recommendations. (1999). Diabetes mellitus and exercise. Diabetes Care, 22, Supp. 1.

28. Dorchy, H. (2002). Sports and type I diabetes: personal experience. Rev. Med. Brux 23, A 211-217.

29. Kelley, D.B. (1996). American Diabetes Association Complete Guide to Diabetes (pp. 88-91, 159-169, 229, 258, 266). Alexandria: VA: American Diabetes Association.

30. Wasserman, D.H. \& Zinman B. (1994). Exercise in individuals IDDM (Technical Review). Diabetes Care 17, 924-937.

31. American Diabetes Association. (2001). Diabetes mellitus and exercise. Med. Sportowa 17(9), 359-364.

32. Guillausseau, P.J. \& Jennings P.E. (Eds.) (2000). Preventing complications in type II diabetes. Gdańsk: VM VIA MEDICA, 32. [in Polish]

33. American Diabetes Association. (2002). Position statement: diabetes mellitus and exercise. Diabetes Care 25 (suppl 1), S64

34. Zinker, B.A. (1999). Nutrition and exercise in individuals with diabetes. Clin. Sports Med. 18(3), 585606.
35. Szczeklik-Kumala, Z., Czech A. \& Taton J. (2000). Tolerance of physical exercise as a factor in determining the use of physical training in people with diabetes. Med. Metaboliczna 7(6), 205-212. [in Polish]

36. Astrand, P.O. \& Ryhming I. (1954). A monogram for calculation of aerobic capacity (physical fitness) from pulse rate during submaximal work. J. Appl. Physiol. 7, 218-221.

37. Peirce, N.S. (1999). Diabetes and exercise. Br. J. Sport Med. 33(3), 161-72; 172-173; 222.

38. Tanaka, H., Monahan K.D. \& Seals D.R. (2001). Agepredicted maximal heart rate revisited. J. Am. Coll. Cardiol. 37, 153-156.

39. Devlin, J.T. \& Ruderman N.B. (Ed.) (1995). Diabetes and exercise: the risk - benefit profile (pp. 3-4). Am. Diabetes Association.

40. Colberg, S.R. \& Swaln D.P. (2000). Exercise and diabetes control. The Physician and Sports Medicine 4, 6367

41. Noczyńska, A. (2009). How to prepare a patient with type I diabetes to treatment with personal insulin pump. Med. Metaboliczna 13(2), 38-44. [in Polish]

42. Maynard, T. (1991). Exercise: Part I Physiological response to exercise in diabetes mellitus. Diabetes Educ. 17, 196-206.

43. Bronus, F. (1993). Nutritional Needs of Athletes. Canada: John Wiley and Sons.

44. Ziemlański, Ś. \& Niedźwiecka-Kącik D. (1997). Nutrition and healthcare recommendations for athletes. Warsaw: Biblioteka Trenera, Centralny Ośrodek Sportu. [in Polish]

Submitted: July 6, 2010

Accepted: September 23, 2010 